\title{
Current Perspectives on the Unique Roles of Exosomes in Drug Resistance of Hepatocellular Carcinoma
}

\author{
Dongdong Xue', Jingzhao Han', Ze Liang', Lin Jia ', Yifan Liu',2, Hongfang Tuo', Yanhui Peng $\mathbb{D}^{\prime}$ \\ 'Department of Hepatobiliary Surgery, Hebei General Hospital, Shijiazhuang, People's Republic of China; ${ }^{2}$ Department of Graduate School, Hebei \\ Medical University, Shijiazhuang, People's Republic of China
}

Correspondence: Yanhui Peng, Department of Hepatobiliary Surgery, Hebei General Hospital, No. 348 Heping West Road, Xinhua District, Shijiazhuang, 05005I, Hebei Province, People’s Republic of China, Tel/Fax +86-3II-859 832I, Email yhpeng@hebmu.edu.cn

\begin{abstract}
As a common malignant tumor worldwide, the prognosis of hepatocellular carcinoma (HCC) remains unsatisfactory, even though treatment methods have improved. Despite the developments in traditional chemotherapy and emerging targeted immunotherapy, the problem of recurrence and metastasis of HCC and adverse effects on survival and prognosis are still serious. Drug resistance is a daunting challenge that impedes HCC treatment. Exosomes, a class of extracellular vesicles ranging in size from 30 to $100 \mathrm{~nm}$, have been the focus of recent studies. Exosomes can activate various signaling pathways and regulate the tumor microenvironment with their cargo, which includes functional lipids, proteins, and nucleic acids. Thus, they change the phenotype of recipient cells via exosome-mediated communication. Exosomes secreted by tumors or stromal cells can also transfer drug-resistant traits to other tumor cells. However, their effects on drug resistance in HCC are not completely understood. In this review, we summarize and discuss the underlying relationship between exosomes and drug resistance in HCC. In addition, we also show that exosomes may act as candidate biomarkers for predicting and monitoring drug responses and as potential targets or vectors to reverse the drug resistance of HCC.

Keywords: hepatocellular carcinoma, exosome, tumor microenvironment, targeted therapy
\end{abstract}

\section{Introduction}

Hepatocellular carcinoma (HCC) is the sixth leading cause of death globally and the third leading cause of cancer-related death. ${ }^{1}$ The five-year overall survival rate of patients with HCC is only approximately $10.1 \%$ due to the difficulty of early diagnosis, rapid progression, high rate of recurrence, and lack of effective treatment. ${ }^{2}$ At present, the treatment methods for HCC are limited, with treatment still based on surgical resection. Consequently, the recurrence rate is as high as $70 \%$ in five years after Surgery, most of which occurs within two years. ${ }^{3}$ Since most HCC patients are diagnosed as latemiddle-stage, survival time can only be prolonged via local radiofrequency ablation, arterial chemoembolization, radiotherapy, and chemotherapy. ${ }^{4}$ Some patients with advanced HCC can benefit from sorafenib, 5-fluorouracil (5-FU), adriamycin, doxorubicin, and platinum drugs. ${ }^{5,6}$ However, the clinical effects of chemotherapy, targeted drugs, and immunotherapy that have emerged in recent years are not satisfactory. One reason is that HCC cells are prone to multiple drug resistance (MDR). ${ }^{6}$ Resistance to chemotherapeutic drugs such as gemcitabine, oxaliplatin, cisplatin, doxorubicin, and 5-FU is the main cause of chemotherapy failure in patients with HCC, especially in patients with advanced HCC or metastasis. ${ }^{7} \mathrm{HCC}$ also develops resistance to multikinase inhibitors, such as sorafenib, and people must choose secondline targeted drugs. ${ }^{8}$ Thus, drug resistance of HCC leads to recurrence and poor therapeutic efficacy. Therefore, it is important to improve the efficacy of anti-tumor drugs by revealing the potential mechanisms of drug resistance in HCC.

Recently, the relationship between HCC and exosomes has become a subject of interest. Exosomes, a type of extracellular vesicle (EV) with a diameter of 30-100 nm, contain various biomolecules, such as proteins and nucleic acid. ${ }^{9}$ Exosomes secreted with lipid bilayers can be taken up by recipient cells via phagocytosis, endocytosis, or 
membrane fusion. The exosomal cargo is then released into the cells, where they perform various physiological functions. ${ }^{10}$ Exosome membrane proteins can also bind to target cell membrane proteins, thereby activating signaling pathways within the target cells and enabling intercellular signaling. ${ }^{11}$ Tumor cells usually secrete more exosomes in response to changes in the microenvironment, such as $\mathrm{pH}$, temperature, and cytotoxic drugs. For example, it was observed that Hep-G2 cells treated with cytotoxic drugs released exosomes containing high-level heat shock proteins (HSPs), which act as triggers for stress response and survival mechanisms. ${ }^{12}$ In addition, exosomes serve a key role in tumor angiogenesis, epithelial-mesenchymal transition (EMT), recurrence, and metastasis. ${ }^{13}$ Evidence shows that exosome-mediated cellular communication is involved in drug resistance. ${ }^{14}$ Exosome studies have led to the proposal of new potential mechanisms of drug resistance. Generally, exosome-related tumor drug resistance is achieved in two ways: first, the exosomes deliver key drug resistance proteins (such as Rab27B) and RNAs (such as miR-31-5p) to other cells, inducing and enhancing their drug resistance; and second, exosomes can engulf drug molecules and excrete them extracellularly, reducing the intracellular drug concentration. ${ }^{15,16}$ In addition, exosomes can fuse with target cells due to their low immunogenicity and high stability, making them an optimal choice as drug carriers. Yukawa et al ${ }^{17}$ proposed that the modification of exosomes led to potential clinical applications. Exosomes are reported to be involved in chemotherapeutic drug resistance, and the inhibition of exosome secretion is effective in sensitizing cancer cells to therapeutic drugs. ${ }^{13}$ In summary, exosomes may be a novel target for HCC therapy.

As a highly heterogeneous disease at the molecular and clinical levels, HCC includes various subtypes with different genetic, pathological and clinical behaviors. ${ }^{18}$ Exosomes mediate multiple drug resistance mechanisms to confer cytochemical resistance in HCC cells (Figure 1), suggesting that exosomes serve an extensive role in the development of HCC. ${ }^{19,20}$ In this review, we summarize the exosomal cargo involved in the drug resistance of HCC (Table 1). In addition, we discussed the role of exosomes as tumor biomarkers and their potential applications in anti-tumor therapy.

\section{Exosomes and Chemoresistance in HCC}

Chemotherapy is one of the treatments for HCC, and the efficacy of drugs depends on the chemosensitivity of cancer cells. However, resistance to anti-tumor drugs remains a major challenge in HCC despite successful advances using targeted therapies. ${ }^{21}$ The emergence of MDR is the main cause of chemotherapy failure in HCC. The mechanisms of drug resistance in HCC are complex and diverse. Drug metabolism, changes in drug targets, DNA damage repair, imbalance between prosurvival and pro-apoptotic factors, activation of survival pathways, adaptation to the tumor microenvironment (TME), and phenotypic transition are all important causes of drug resistance. ${ }^{22}$ It is increasingly recognized that exosomes, secreted by tumor cells and possessing special transport capacity, are involved in multiple links of drug resistance, resulting in the transfer of drug-resistant phenotypes between tumor cells and non-tumor cells in $\mathrm{HCC} .{ }^{23}$ As crucial intercellular carriers, the secretion of exosomes is a rate-limiting step, rather than endocytosis of recipient cells. ${ }^{12}$ This means that interfering with exosome
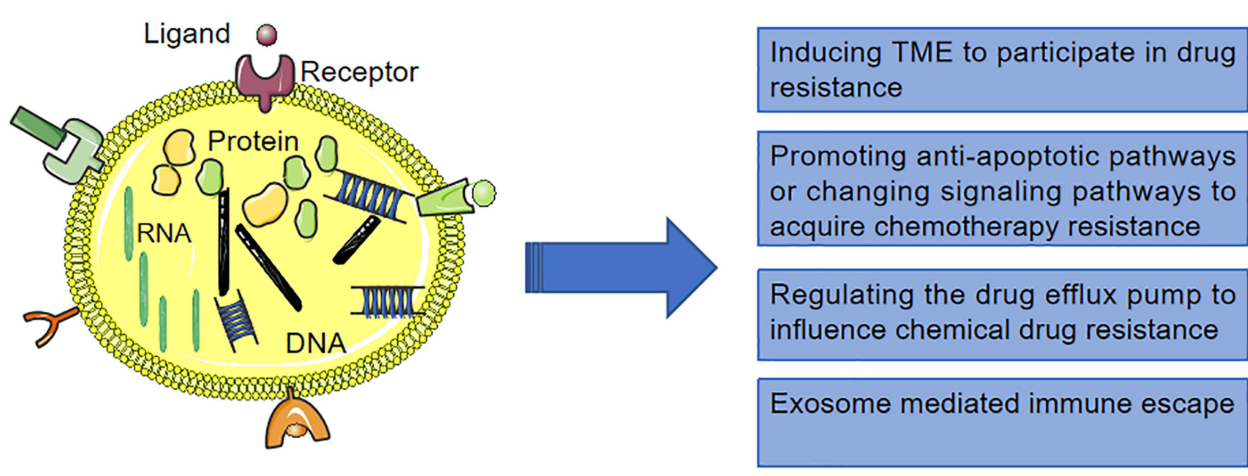

Exosomes harbor proteins, mRNAs, miRNAs, IncRNAs, circRNAs, and DNAs

Figure I Exosomes mediate multiple drug resistance mechanisms to confer cytochemical resistance in $\mathrm{HCC}$ cells. 
Table I Exosomal Cargoes Involved in Drug Resistance of Hepatocellular Carcinoma

\begin{tabular}{|c|c|c|c|}
\hline Classification & Cargoes & Effects & Reference \\
\hline \multirow[t]{10}{*}{ IncRNAs } & lincVLDLR & Increase the expression of $A B C G 2$ in recipient cells & [36] \\
\hline & linc-ROR & Reduced chemotherapy-induced cell death in recipient cells & {$[4 I]$} \\
\hline & lincRNA- & Increased expression of $A B C G 2$ & [36] \\
\hline & LVVDL & & \\
\hline & lincRNA & Induced expression of $A B C B I$ and $A B C G 2$ & [112] \\
\hline & HOTAIR & & \\
\hline & lincRNA & Up-regulated miR-7-5p-ABCCI & [113] \\
\hline & KCNQIOTI & & \\
\hline & IncRNA CTD- & Regulate chemoresistance related genes & {$[115]$} \\
\hline & $2116 \mathrm{~N} 20.1$ & & \\
\hline \multirow[t]{3}{*}{ circRNAs } & circNFIX & Increases the expression of $A B C G 2$ via the circNFIX-miR-I 32 axis & [37] \\
\hline & circUHRFI & Up-regulated the expression of TIM-3, induce NK cell dysfunction. & [54] \\
\hline & circFoxo3 & Up-regulated miR-199a-5p-ABCCI & [114] \\
\hline \multirow[t]{18}{*}{ miRNAs } & miR-32-5p & Activate the PI3K/Akt pathway and regulated angiogenesis and EMT & [39] \\
\hline & miR-2I & Down-regulate the expression of tumor suppressor genes PTEN and PTENpI & [40] \\
\hline & $\operatorname{miR}-214$ & Reduce cell vitality, inhibits migration, and promotes apoptosis in vitro & [45] \\
\hline & miR-199a-3p & Reverse the drug resistance of $\mathrm{HCC}$ to cisplatin & [46] \\
\hline & $\mathrm{miR}-122$ & Render cancer cells sensitive to chemotherapeutic agents & [47] \\
\hline & miR-I46a & Promote M2 polarization and inhibit the anti-HCC function of $\mathrm{T}$ cells & [53] \\
\hline & miR-23a-3p & $\begin{array}{l}\text { Activation and upregulation of PD-LI via PI3K-AKT pathway, induced immune escape } \\
\text { mechanism via impairing the function of CD8+T cells }\end{array}$ & [56] \\
\hline & miR-I0b & Promoted the proliferation, migration and invasion of HCC cells & [65] \\
\hline & miR-1247-3p & Convert normal fibroblasts to CAFs & [7I] \\
\hline & $\operatorname{miR}-210$ & Change cellular expression levels and paracrine or juxtacrine signaling & [76] \\
\hline & miR-27a-3p & Enhance the activity of $\mathrm{HCC}$ stem cells and promote drug resistance & [77] \\
\hline & miR-I55 & Promote EMT and mediate drug resistance & [83] \\
\hline & miR-128-3p & Suppressing Bmi-I expression & [86] \\
\hline & miR-222 & PTEN inhibition, p27/kip inhibition & [107] \\
\hline & miR-49I-3p & Induce $A B C B I$ expression & [108] \\
\hline & miR-34a & Down-regulated $A B C B I$ & [109] \\
\hline & miR-133a & Down-regulated $A B C C I$ & {$[110]$} \\
\hline & miR-I83 & Up-regulated HIF-I $\alpha-A B C B \mid, A B C C 2$ & {$[111]$} \\
\hline \multirow[t]{2}{*}{ Protein } & HMGBI & Promote the expansion of TIM-I+Breg cell & [55] \\
\hline & Heat shock & Enhance the cytotoxicity and granzyme B production of NK cells & {$[6 I]$} \\
\hline
\end{tabular}

Abbreviations: IncRNAs, long noncoding RNAs; HCC, hepatocellular carcinoma; EMT, epithelial-mesenchymal transition; PD-I, programmed death-I; PTEN, phosphatase and tensin homolog; $A B C$, adenosine triphosphate binding cassette.

secretion is an important mechanism for regulating the transfer of cargo between cells. The exosome-mediated chemoresistance of resistant and sensitive HCC cells is shown in Figure 2.

\section{Exosome-Mediated Drug Efflux and Inactivation}

The accumulation of cytotoxic drugs in cancer cells is a prerequisite for drug efficacy. Therefore, the increase in drug efflux from cancer cells is a key factor leading to drug resistance in tumor cells. Exosome-mediated direct drug efflux serves a key role that cannot be ignored. ${ }^{24}$ As early as 2003 , Shedden et $a^{25}$ reported that gene expression associated with vesicle shedding correlated with chemosensitivity profiles, and their observations implicated vesicle shedding as a drug efflux mechanism potentially involved in drug resistance. Safaei $\mathrm{et} \mathrm{al}^{26}$ found that exosomes secreted from cisplatinresistant tumor cells contained 2.6-fold more platinum than those secreted from cisplatin-sensitive cells. A recent study led by $\mathrm{Jin}^{12}$ indicated that chemotherapeutic agents could be transferred into exosomes, stimulating exosome production or release, thus reducing drug bioactivity. They found that MCF7 cells (breast cancer cell line) with low P-glycoprotein 


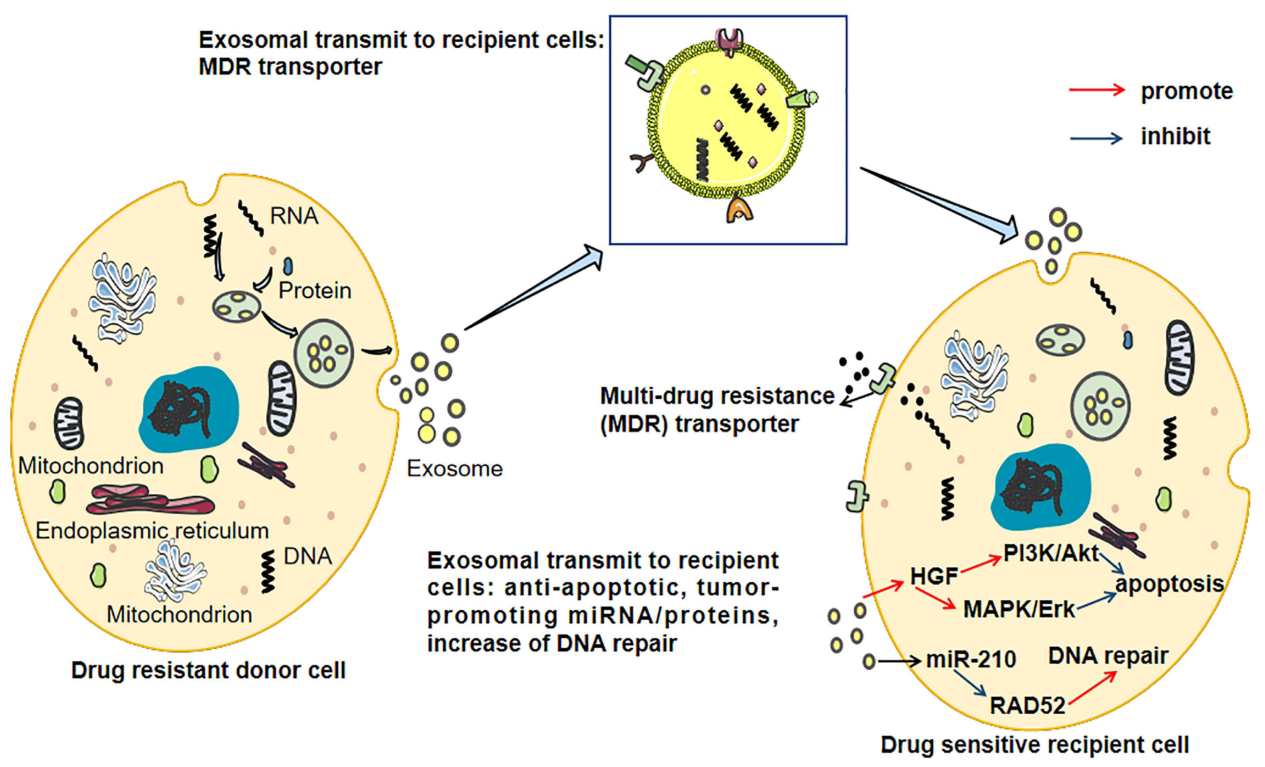

Figure 2 The exosome-mediated chemoresistance between resistant and sensitive HCC cells. Exosomes transfer multi-drug resistance (MDR) transporters from drugresistant cells to sensitive cells causing increased efflux of cytotoxic drugs via integrating MDR transporters on the cell membrane. Besides, exosomes induce increased antiapoptotic signaling and DNA repair in the recipient cells.

(P-gp) levels reduced the intracellular concentration of paclitaxel by up to $80 \%$ via exosome-efflux. This phenomenon suggests a potential mechanism for chemoresistance.

As a membrane transporter, $\mathrm{ABCB} 1$ (also known as P-gp), belonging to the ATP-binding cassette ( $\mathrm{ABC}$ ) family, is the most prominent factor in the development of MDR. ${ }^{15} \mathrm{ABCB} 1$ is expressed in more than $50 \%$ of cancers exhibiting MDR and is associated with the induction of chemotherapy. ${ }^{27}$ Another study showed that ABCB1 could transport about 20 different cytotoxic drugs, including paclitaxel and doxorubicin. ${ }^{28}$ Tadashi et al ${ }^{29}$ investigated organic cation transporter (OCT)-1 and OCT3, as well as the ABC family efflux transporters MDR1/ ABCB1, multidrug resistance protein (MRP) 2, and breast cancer resistance protein (BCRP/ABCG2) and showed that the expression of transporters (particularly BCRP) is highly variable. This study shows the importance and variability of membrane transporters in intracellular and extracellular drug transport. Although a variety of MDR pump inhibitors (such as probenecid) have been developed, their clinical efficacy is unsatisfactory. ${ }^{30}$ Is it possible that exosomes affect the distribution of membrane transport proteins between drug-resistant and sensitive cells, thereby inducing the production of drug-resistant proteins? There is already evidence showing that exosomes can transport drug transporters such as ABCB1 from drug-resistant cells to sensitive cells, which in turn can lead to drug resistance in sensitive cells. ${ }^{27}$ Short exposure to chemotherapeutic drugs can significantly increase the release of ABCB1-containing EVs from Rab8B-mediated drug-resistant cells, and accelerate the fusion of these EVs with the plasma membrane of sensitive tumor cells by downregulating Rab5. This causes a significant enhancement of intercellular ABCB1 metastasis, thus conferring drug-resistant phenotype to sensitive cancer cells. ${ }^{31}$ Tang et al $^{7}$ confirmed that exosomes from HepG2 cisplatin-resistant cells could transfer ABCB1 to various HCCsensitive cells, including SMMC-7721 and Huh7 cells. Other efflux transporters, such as ABCB2, ABCA3, and MRP1, can also regulate drug resistance ${ }^{32,33}$ via exosome transport. These findings provide a novel molecular mechanism for how chemotherapeutic drugs help sensitive cancer cells acquire drug resistance.

Exosomal non-coding RNAs (ncRNAs), including micro RNAs (miRNAs), long non-coding RNAs (lncRNAs), and circular RNAs (circRNAs), are dysregulated in drug-resistant cancer cells and regulate the expression of ABC transporters via various mechanisms. ${ }^{34}$ Borel et $\mathrm{al}^{35}$ concluded that the upregulation of $\mathrm{ABC}$ transporters in $\mathrm{HCC}$ is related to the downregulation of miRNA. A study revealed that the $\mathrm{ABC}$ transporter was regulated by exosomal lncRNA, and found an increase in lincVLDLR expression in exosomes released by HCC cells, which transmitted resistance via increasing the expression of ABCG2 in recipient cells. ${ }^{36}$ The overexpression of exosomal circNFIX increases the expression of ABCG2 via the circNFIX-miR-132 axis, thereby promoting the resistance of glioma cells to temozolomide (TMZ). The serum 
exosomal circNFIX in patients with TMZ resistance increased and was associated with their prognosis. ${ }^{37}$ In summary, studying ncRNAs in exosomes associated with $\mathrm{ABC}$ transport proteins and revealing their biological function in MDR may provide a novel theoretical basis for reversing this phenotype.

\section{Exosome-Mediated Transfer of Pro-Survival Cargo}

Cancer cells contain more exosomes than normal cells do. More importantly, the cargo of exosomes derived from cancer cells is distinct. For example, exosomes from cancer cells contain more miRNAs, which are closely associated with cancer invasion and drug resistance, than exosomes from normal cells. ${ }^{38}$ A study showed that exosomes from different HCC cell lines contained various proteins and tumor-promoting RNAs. ${ }^{9}$ Exosome-mediated cargo transfer is regulated by various cytokines,

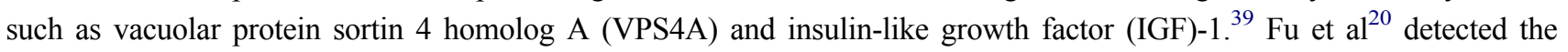
expression of miR-32-5p and phosphatase and tensin homolog (PTEN; a tumor suppressor gene) in multidrug resistant cell lines (Bel/5-FU) and sensitive cell lines (Bel7402) via real-time polymerase chain reaction. The results demonstrated that Bel/ 5-FU transmitted miR-32-5p to Bel7402 through exosomes, which activated the PI3K/Akt pathway and regulated angiogenesis and EMT to further induce multidrug resistance. Cao et $\mathrm{al}^{40}$ confirmed that exosomal miR-21 could downregulate the expression of tumor suppressor genes PTEN and PTENp1 (PTEN pseudogene 1), and affect the development of HCC. Recently, lncRNAs carried by exosomes have attracted increasing attention. Exosome-mediated lncRNA transport, especially linc-VLDLR and linc-ROR, promotes chemotherapy resistance in HCC. ${ }^{39}$ Takahashi et $\mathrm{al}^{36}$ found that linc-VLDLR was highly expressed in exosomes from malignant liver tumors, and regulated the drug resistance of recipient cancer cells to antitumor drugs such as sorafenib, camptothecin, and doxorubicin. Knockout of the linc-VLDLR gene reduces the viability of cancer cells and inhibits cell cycle processes. During chemotherapeutic stress, tumor growth factor (TGF)- $\beta$ increases the expression of $\mathrm{CD} 133+$ cells partly due to the high expression of exosomal linc-ROR, which generally increases the drug resistance of HCC to chemotherapy. Considering the functional role of linc-ROR in TGF- $\beta$-dependent chemotherapy resistance, the use of siRNA to knockout linc-ROR to enhance chemotherapy-induced apoptosis and cytotoxicity may be a potential method for HCC treatment. ${ }^{41}$ Based on high-throughput sequencing, increasing evidence has shown that circRNAs are also involved in various cancer-related processes, including tumor initiation, progression, relapse, and drug resistance. ${ }^{42}$ $\mathrm{Xu}$ et $\mathrm{al}^{43}$ found that exosomal circRNAs play a role in preventing the Pre-MRNA Processing Factor 19-mediated degradation of Y box-binding protein 1 (YB-1), thus affecting the expression of downstream gene targets of YB-1, including AKT, Raf1, ERK, c-Myc, and TGF- $\beta$. These exosomal RNAs also enabled the transmission of sorafenib resistance among HCC cells. These studies suggest that exosomal ncRNAs play a broad role, and blocking their functional pathways may be one of the research directions for improving the drug resistance of $\mathrm{HCC}$ in the future.

Exosomes also regulate drug resistance via transmitting cytokines. $\mathrm{It}^{44}$ has been confirmed that exosomes from HCC induce sorafenib resistance via increasing the level of hepatocyte growth factor (HGF) in the TME and activating the c-Met/Akt pathway. This suggests that HGF/c-Met may be a vital target for improving sorafenib resistance in HCC. Exosomes from highly invasive tumors may lead to stronger drug resistance. ${ }^{44}$

However, not all exosomal cargo promotes drug resistance. When exosomes rich in miR-214 fuse with cells, they reduce cell vitality, inhibit migration, and promote apoptosis in vitro. Exosomal miR-214 can also reverse cisplatin resistance of gastric cancer, which may be a potential alternative to cisplatin-refractory gastric cancer. ${ }^{45} \mathrm{Zhang}$ et ${ }^{46}$ verified that exosomal miR-199a-3p (exo-miR-199a-3p) could sensitize HCC cells to cisplatin via flow cytometry, cell counting Kit-8 (CCK-8) assay, immunofluorescence assay, and transwell assay. Another research showed that adipose tissue-derived mesenchymal stem cells (AMSCs) transfected with miR-122 could package miR-122 into secreted exosomes, which mediated the transmission of miR-122 between AMSCs and HCC cells, thereby rendering HCC cells sensitive to chemotherapeutic agents via alteration of miR-122-target gene expression. ${ }^{47}$ These studies indicate that the functions of exosomal cargo are diverse. This poses a challenge to the use of exosomes as vehicles for antitumor drugs.

\section{Exosomes and Immunotherapeutic Resistance in HCC}

Compared with other cancers, HCC is less sensitive to traditional chemotherapy. ${ }^{48}$ In recent years, immunotherapy has gained popularity, and a variety of drugs, such as atezolizumab, nivolumab, and pembrolizumab, have been approved for 
HCC patients. ${ }^{49}$ Although immunotherapy has exhibited efficacy in the treatment of HCC, some immunotherapies, such as checkpoint inhibitors, have exhibited limited benefit for HCC patients. ${ }^{50}$ It has been found that multiple immune checkpoints, including programmed death-ligand-1/programmed death-1 (PD-L1/PD-1), can significantly affect cancer development. PD-L1/PD-1 inhibitors have been widely used in clinics, but they do not exhibit the expected therapeutic advantages. ${ }^{51}$ This may be explained by variation in types of drug resistance. Immunosuppression and immune tolerance are key factors in the occurrence, development and metastasis of HCC. The unique immunobiology of the liver promotes tumorigenesis and tumor immune tolerance under conditions of fibrosis and chronic inflammation. ${ }^{52}$ Exosomes serve multiple roles in the interaction between HCC cells and immune cells, thereby mediating tumor progression and immune evasion. ${ }^{53}$ It has been proven that the exosomes secreted by drug-resistant tumor cells can reshape immune cells (T cells, macrophages, and dendritic cells) in the TME, disrupting their function and preventing them from inducing host immune responses. ${ }^{16}$ The role of exosomes in immunotherapeutic resistance can not be ignored. Zhang et $\mathrm{al}^{54}$ have found that circular ubiquitin-like with PHD and ring finger domain 1 RNA (circUHRF1) was highly expressed in HCC tissues and exosomes derived from HCC. In addition, this study demonstrated that exosomal circUHRF1 was delivered into natural killer (NK) cells and upregulated the expression of $\mathrm{T}$ cell immunoglobulin and mucin domain 3 (TIM-3), thereby inducing NK cell dysfunction. More importantly, HCC cells overexpressing circUHRF1 present the characteristics of anti-PD-1 therapy. Ye et $\mathrm{al}^{55}$ showed that exosome-derived high mobility group box-1 protein (HMGB1) could promote the expansion of TIM-1+Breg cells, which mediate immune escape in HCC via the Toll-like receptor (TLR) 2/4 and mitogen-activated protein kinase (MAPK) signaling pathways, suggesting that the inhibition of exosomal HMGB1TLR2/4-MAPK pathways could treat this immune tolerance feature of HCC. Han et al ${ }^{53}$ revealed that exosomal miR146a could promote $\mathrm{M} 2$ polarization and inhibit the anti-HCC function of T cells. It has been reported that endoplasmic reticulum (ER) stress causes HCC cells to release miR-23a-3p-rich exosomes. Once internalized by M2 cells, miR-23a$3 p$ suppresses the expression of PTEN, resulting in the activation and upregulation of PD-L1 via the PI3K-AKT pathway, which induces an immune evasion mechanism via impairing the function of CD8+T cells. ${ }^{56}$ These findings provide a novel alternative to overcome immunosuppression and immune evasion in the TME of HCC.

In addition, PD-L1 can be anchored on the surface of exosomes as a transmembrane protein and plays a vital role in immune evasion and drug resistance in immunotherapy. Exosomal PD-L1 imparts an immunosuppressive phenotype via three main mechanisms: direct endogenous exosomal PD-L1, indirect induced PD-L1, and PD-L1-mediated antagonism of immune checkpoint blockade therapy change. ${ }^{57}$ Exosomal PD-L1 can inhibit $\mathrm{T}$ cell function and induce systemic immunosuppression. ${ }^{58}$ Chen et $\mathrm{al}^{59}$ revealed that interferon- $\gamma$ (IFN- $\gamma$ ) could increase the level of PD-L1 on exosomes, thereby inhibiting the function of CD8+ T-cells and promoting tumor growth. Samantha et $\mathrm{al}^{57}$ also found that the level of PD-L1 expression on exosomes is often positively correlated with the severity of cancer staging and negatively correlated with survival in a wide array of cancers, such as breast cancer and colon cancer. Conversely, tumor-derived exosomes can also express a large number of major histocompatibility complex class I molecules (MHC I) and tumor markers, such as HSP, participating in antigen presentation and T cell stimulation, to trigger a CD8+ T-cell-dependent anti-tumor response ${ }^{60} \mathrm{HCC}$ cell-derived HSP-bearing exosomes can effectively enhance the cytotoxicity and granzyme $\mathrm{B}$ production of NK cells, upregulate the expression of inhibitory receptor CD94, and downregulate the expression of activating receptors CD69, NKG2D, and NKp44. Notably, anti-tumor drugs that cells are resistant to enhance exosome release and produce more exosome-carrying HSPs, thus enhancing the cytotoxic response. ${ }^{61}$ These studies, which arrive at contradictory conclusions, show that the functions of exosomal cargos and membrane modifiers are complex and diverse, and that targeting a single cargo cannot achieve satisfactory therapeutic effects. Further research is needed to verify whether HCC-derived exosomes affect other immune factors. The exact mechanisms underlying the immunomodulatory effects in HCC remain to be elucidated and are promising targets for future research.

\section{Exosomes Regulate TME in the Drug Resistance of HCC}

Tumor heterogeneity, recurrence, metastasis, and drug resistance are the main reasons why tumors are difficult to treat. The therapeutic efficacy of anti-tumor drugs is unsatisfactory due to insufficient targeting, severe side effects of drugs and radiotherapy, and many other challenges. ${ }^{62}$ This suggests that tumor treatment should not only target malignant tumor cells but also consider the effect of the TME. TME includes tumor epithelium and various tumor-supporting cells, 
such as immune cells, fibroblasts, immunosuppressive cells, adipocytes, endothelial cells, and various cytokines. The interaction between tumor cells and non-tumor cells in the TME is triggered via direct cell-to-cell contact and the transfer of soluble molecules (such as cytokines and chemokines), while exosomes play a key role in intercellular molecular transfer. ${ }^{63}$ In addition, the interaction between TME and tumor cells is a key contributor to immune escape, recurrence, and metastasis. ${ }^{64}$ An in-depth study of the complex exosome-mediated intercellular communication network is essential for elucidating the mechanisms that promote tumor cell growth and drug resistance. TME can also affect the release and function of exosomes. Tian et $\mathrm{al}^{65}$ demonstrated that an acidic microenvironment triggered the activation of HIF-1/2 $\alpha$ and stimulated the expression of exosomal miR-21 and miR-10b, which significantly promoted the proliferation, migration and invasion of HCC cells in vivo and in vitro. Here, we summarize the latest studies to better illustrate the role of exosome and TME interactions in HCC resistance.

The impaired function of stromal cells contributes to the maintenance of cancer cells and creates a favorable TME for the growth of cancer cells. ${ }^{66}$ Exosomes secreted by mesenchymal stem cells (MSCs) promote the progression of breast cancer via inducing the differentiation of monocytic myeloid-derived suppressor cells into highly immunosuppressive M2-polarized macrophages. ${ }^{67}$ Zhang et al $^{68}$ reported that MSC-derived exosomes enhanced CD4+CD25+ T-cells or CD4 $+\mathrm{CD} 25+$ Foxp3 + Tregs cells in vitro and in vivo via allogeneic antigen-presenting cell (APC)-mediated pathways. Similarly, tumor-derived exosomes are involved in the differentiation of fibroblasts to cancer-associated fibroblasts (CAFs), which promotes the occurrence of tumor drug resistance. ${ }^{69}$ For example, exosomes isolated from HCC cells exhibit a great capacity to convert normal HSCs to CAFs, because exosomal miR-21 directly targets PTEN, leading to the activation of PDK1/AKT signaling in HSCs. In addition, activated CAFs further promote tumor progression via secreting angiogenic cytokines, including VEGF, MMP2, MMP9, and TGF- $\beta .^{70}$ High-metastatic HCC cells, which can secrete exosomes with miR-1247-3p, exhibit a greater capacity to convert normal fibroblasts to CAFs than low-metastatic HCC cells, leading to sorafenib resistance. ${ }^{71}$ Exosomes secreted by CAFs can also develop drug resistance. Cisplatin and paclitaxel promote secretion of miR-522 from CAFs via activating the ubiquitin-specific protease 7/heterogeneous nuclear ribonucleoprotein A1 axis, resulting in the inhibition of chiradonate lipoxygenase 15 and decreased lipid

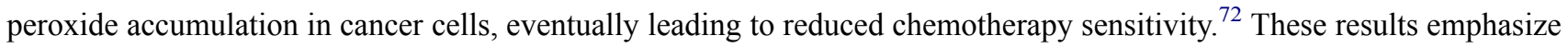
the importance of studying the mechanisms of drug resistance induced by stromal cell exosomes.

Cancer stem cells (CSCs) are a subgroup of cancer cells with functions similar to those of normal stem cells, and they have the potential for self-renewal, unlimited proliferation, and differentiation. In addition, CSCs possess the ability to evade immune surveillance, which is closely related to tumor invasion, metastasis, drug resistance and recurrence after treatment. $^{73}$ CSCs can activate non-tumor cells through exosomes. Li et al $^{74}$ highlight that CSCs from esophageal squamous cell cancer secreted exosomes containing fragile X mental retardation 1-antisense RNA 1 and transfer the stem cell phenotype to recipient non-CSCs via activation of TLR7-NFאB signaling and upregulating c-Myc levels in recipient cells. CSCs exhibit chemoresistance or radioresistance due to EMT and DNA damage checkpoint activation. ${ }^{75}$ Yang et $^{7}{ }^{76}$ suggested that the transfer of CSCs' exosomal miRNAs (such as miR-210) could change cellular expression levels and paracrine or juxtacrine signaling, thus preventing the goals of chemotherapy, such as cell cycle arrest and cancer cell apoptosis. Similarly, M2 macrophage-derived exosomes with miR-27a-3p can enhance the activity of HCC stem cells and promote drug resistance. ${ }^{77}$ Therefore, specific targeted therapy of tumor stem cells and their exosomes in the TME may improve the efficacy of anti-tumor therapy.

EMT is considered to be the process by which epithelial cells lose their epithelial phenotype (such as E-cadherin expression) and acquire more mesenchymal phenotypes (such as N-cadherin expression). ${ }^{78}$ Tumor cells acquire mobility and invasiveness through EMT, which is the initial step in distant metastasis of cancer. ${ }^{79}$ In addition, the emergence of CSCs is

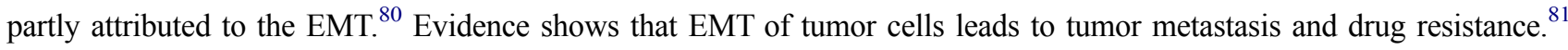
Several important molecules, such as transcription factors (SNAIL, TWIST, and ZEB) and miRNA (miRNA-200 family, miR15, and miR-186), which are involved in EMT and drug resistance, are considered vital. However, the connection between EMT and drug resistance has not been fully elucidated. ${ }^{82}$ Santos et al ${ }^{83}$ analyzed the transfer ability of drug-resistant phenotypes to sensitive cells in breast cancer via MTT assay and scratch test. The results showed that exosomes could

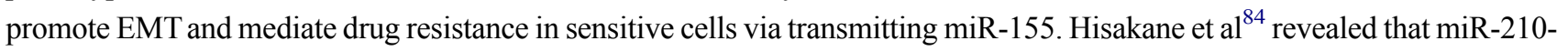
$3 p$ was upregulated in exosomes isolated from osimertinib-resistant HCC827, and their study found that exosomal miR-210- 
3p may promote EMT and induce resistance to osimertinib in HCC827 cells (a type of lung cancer cell line). Conversely, Kim et $\mathrm{al}^{85}$ revealed that exosome-mediated miR-125b transfer disrupted TGF- $\beta 1$-induced EMT and TGF- $\beta 1 /$ SMAD signaling pathway in recipient cells via decreasing SMAD2 protein expression. In addition, exosomal miR-125b expression was downregulated after metastasis. Liu et $\mathrm{al}^{86}$ developed a protocol for exosome-mediated miR-128-3p delivery to oxaliplatinresistant cells in colorectal cancer. On one hand, exosomal miR-128-3p suppressed EMT via suppressing Bmi-1 expression. On the other hand, exosomal miR-128-3p reduced the efflux of oxaliplatin via inhibiting the expression of drug transporter MRP5, thus increasing intracellular oxaliplatin accumulation. Thus, exosomes can be used as a novel strategy to enhance the sensitivity to chemotherapy. In conclusion, the interaction between exosomes and TME is complex and needs to be explored constantly to provide a new orientation for overcoming drug resistance in $\mathrm{HCC}$ in the future. Here we summarize that, in HCC cells, exosomes, as carriers of information, mediate communication between the various cell types in TME leading to the acquisition of drug resistance (Figure 3).

\section{Potential Clinical Applications of Exosomes in HCC Drug Resistance Exosomes as Biomarkers on the Prediction of Therapeutic Response in HCC}

Biomarkers can be used to predict tumor response to specific treatments and represent sensitivity or resistance to antitumor drugs. ${ }^{87}$ Biomarkers for predicting treatment response in $\mathrm{HCC}$ have been widely studied. Yu et al ${ }^{88}$ analyzed the transcriptional dataset of $225 \mathrm{HCC}$ tissues and 220 non-HCC liver tissues from the Gene Expression Omnibus database. It was revealed that MTR4 (an RNA helicase) is overexpressed in a few HCC tissues and is an independent diagnostic marker for predicting poor prognosis in patients with HCC. Myojin et al ${ }^{89}$ indicated that ST6GAL1, a tumor-derived secreted protein positively regulated by FGF19, is a tumor biomarker in lenvatinib-susceptible HCC. Blood-based liquid biopsy is a good method for evaluating treatment response due to its minimal invasiveness, reproducibility, and ability to dynamically evaluate specific molecular markers.

With the understanding of exosome biology and its correlation with tumor drug resistance, the unique characteristics of exosomes (surface markers, cargo, relatively stable structure and ubiquity in body fluids) and exosomal cargo

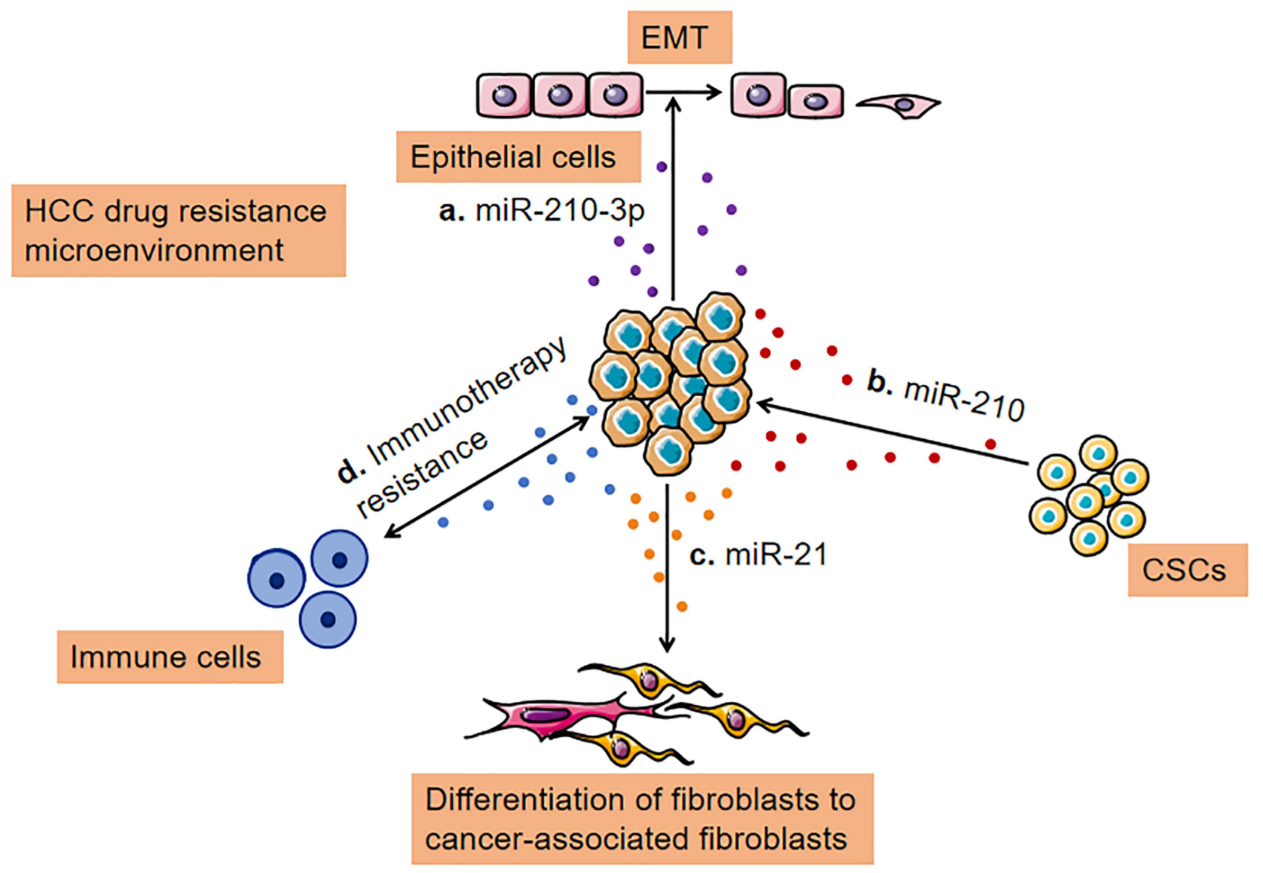

Figure 3 Transfer of drug resistance via exosomes between TME and HCC cells. (A) Exosomes loaded with a high level of miR-2I0-3p lead to epithelial-mesenchymal transition; (B) Cancer cells release miRNA (miR-210) containing exosomes to stimulate CSC-like features; (C). Exosomes secreted by cancer cells can promote cancerassociated differentiation in adjacent fibroblasts polarization towards a tumor-supportive phenotype; (D) Exosomes derived from cancer cells, immune cells, and other stromal cells serve essential roles in remodeling the tumor immune microenvironment.

Abbreviation: CSC, cancer stem cell. 
constantly change with disease conditions, ${ }^{90}$ making them attractive as potential biomarkers. On the one hand, exosomes protect molecules from degradation. Compared with circulating tumor RNAs or DNAs, the nucleic acids contained in exosomes may be more informative and reproducible. Exosomes, on the other hand, can be used to obtain information via quantification of the DNA, RNA, and proteins they carry. ${ }^{24}$ Theoretically, these studies provide more accurate information for predicting the treatment response of HCC. Kim et $\mathrm{al}^{85}$ believed that exosomal miR-125b may be a reliable biomarker for the diagnosis and evaluation of extrahepatic metastasis of HCC. Zhu et al ${ }^{91}$ established a prognosis and recurrence risk prediction model, which presented a significant predictive performance, based on two exosomal genes (MYL6B and THOC2). These findings provide a foundation for improving the early detection of HCC via identifying molecular markers. However, the application of exosomes as biomarkers remains a challenge because of the mixing of tumor-derived exosomes and normal cell-derived exosomes in the circulation. ${ }^{92}$

\section{Exosomes as Novel Therapeutic Interventions in HCC Drug Resistance}

The efficacy of anti-tumor drugs is limited due to poor drug solubility, short half-life, and inefficient drug delivery, leading to drug resistance and significant systemic toxicity. ${ }^{16}$ In addition, the low efficiency of drug delivery is a vital reason for treatment failure. The drug encounters a variety of obstacles before it reaches and acts on the intended target after entering the circulation through intravenous injection. ${ }^{12}$ To improve the chemotherapeutic efficacy of HCC, there is an urgent need to develop a drug delivery system exhibiting active targeting and achieve local, controllable, and continuous drug release. Exosomes exhibit excellent histocompatibility and engineered exosomes can specifically target tumor tissues, improve the distribution of drugs in the body, improve efficacy, protect normal cells, and minimize toxicity. ${ }^{93}$ Due to the heterogeneity of exosomes from different cells, the current technology to treat tumors via exosomes is not that precise or effective, and various obstacles still need to be overcome. ${ }^{94}$ The selection of appropriate exosomes carrying biomolecules or chemical drugs for HCC and how to effectively modify and transfer exosomes to recipient cells are of great significance. Various experiments have confirmed that the assembly of tumor cell-targeting ligands (such as peptides, antibodies, and lipids) on the surface of drug-loaded exosomes can achieve tumor-specific drug delivery and reduce non-specific toxicity. ${ }^{95,96}$ Lin et al $^{97}$ applied iRGD-modified (iRGD: a 9-amino acid cyclic peptide) exosomes with siCPT1A, a key enzyme of fatty acid oxidation (FAO), to specifically transport siCPT1A into colon cancer cells to suppress FAO, which plays a vital role in drug resistance in cancer cells. Consequently, iRGD-modified exosomes significantly inhibited CPT1A in cancer tissues and reversed oxaliplatin resistance via inhibiting FAO with high safety. Lima et $\mathrm{a}^{98}$ found that cytokines in TME, especially CCL2, modify cancer exosomes via binding to surface glycosaminoglycan side chains of proteoglycans, resulting in the accumulation of exosomes in specific cell subgroups and organs. The accumulation of exosomes leads to changes in the immune environment of these organs. Notably, CCL2modified exosomes directly express CCL2 receptor CCR2, suggesting that exosome-binding cytokines are the key determinants of exosome-cell interaction. These studies also provide a reasonable strategy for the application of exosomes in the treatment of HCC. A variety of receptors and proteins, such as glypican-3 (GPC3), somatostatin receptor (SSTR), and a cluster of differentiation 44 (CD44), are relatively highly expressed on the surface of liver cancer cells and normal liver cells. Exosomes incorporated with specific antibodies targeting these proteins may help to develop an effective therapy for advanced HCC. ${ }^{99}$ In addition to exosomes, studies have focused on the development of exosomal analogs for drug delivery. ${ }^{100,101}$ Liu et al ${ }^{102}$ developed a drug nanocarrier for targeted chemotherapy of HCC. Structurally, the homotypic HepG2 cell membrane was used as the cloak, and a poly lactic-co-glycolic acid (PLGA) nanoparticle as the core, manufacturing the nanocarrier HepM-PLGA. Doxorubicin carried by HepM-PLGA nanoparticles exhibited high delivery efficiency and remarkable therapeutic effects in vitro and in vivo. It provides a promising nano-targeted drug delivery system for drug-resistant HCC. However, targeted delivery applications based on HCC cell surface proteins are rare, and there should be studies on antigen-directed exosome delivery.

In this promising new era of cancer immunotherapy, cancer vaccination is the ultimate goal. ${ }^{103}$ The latest research shows that exosomes secreted by HCC antigen-modified dendritic cells (DCs) can be used as cell-free vaccines. The findings of Lu et al ${ }^{104}$ proved that AFP-enriched dendritic cell-derived exosomes (Dexs) could trigger effective antigenspecific anti-tumor immune responses and reshape the TME of HCC, as demonstrated by significantly increased $\gamma$-IFNexpressing CD8+T lymphocytes, elevated levels of $\gamma$-IFN and IL-2, and fewer CD25+Foxp3+Tregs and decreased levels 
of IL-10 and transforming growth factor- $\beta$ in tumors. Li et al ${ }^{105}$ revealed that Dexs loaded with recombinant adenoassociated viral vector (rAAV)-carrying AFP gene (rAAV/AFP) can effectively stimulate the proliferation of $\mathrm{T}$ cells and induce the activation of $\mathrm{T}$ cells into antigen-specific cytotoxic $\mathrm{T}$ lymphocytes (CTL) exhibiting an immune response against liver cancer. In addition, it seemed that Dex-sensitized DC precursors could trigger MHC I-restricted CTL responses more effectively, maximizing specific immune responses against HCC. These studies show that Dexs, which present the advantages of dendritic cells and cell-free vectors, can partially replace mature DCs as tumor vaccines or natural anti-tumor adjuvants. It provides a novel alternative for HCC therapy.

\section{Conclusions and Perspective}

Cancer is not only a large number of malignant cells, but also a complex "rogue" organ, in which different types of cells, such as fibroblasts, adipocytes, immune cells, and tumor vascular system cells, are involved in tumor homeostasis. ${ }^{106}$ Anti-tumor drug resistance caused by a series of physiological activities has been an obstacle to cancer treatment for a long time. Although significant progress has been made in modern anti-tumor drugs, the occurrence of drug resistance often leads to the failure of HCC treatment. In addition to existing chemotherapy and emerging immunotherapy, there is an urgent need to develop new methods to eliminate tumor progression and recurrence caused by drug resistance. To explore effective new treatments, we must study the role of tumors and their microenvironment, including lymphocytes, macrophages, myeloid suppressor cells, and their exosomes in drug resistance. Exosomes have the unique potential to capture the dynamic complexity of cancer and can be used to test a variety of biological components associated with HCC resistance in real-time. At present, our understanding of exosome physiology, release, transport, internalization, and transport mechanisms is limited, and the mechanism of interaction and modification between exosomes and recipient cells also requires more comprehensive research. ${ }^{53}$ Even though there are various challenges, exosomes may be used as a candidate biomarker to predict and monitor the efficacy of treatment in patients with HCC, and as a potential target or vector to reverse drug resistance, which serves a vital role in tumor detection, prediction and treatment in the future.

\section{Disclosure}

The authors report no conflicts of interest in this work.

\section{References}

1. Dong G, Zhang S, Shen S, et al. SPATS2, negatively regulated by miR-145-5p, promotes hepatocellular carcinoma progression through regulating cell cycle. Cell Death Dis. 2020;11(10):837. doi:10.1038/s41419-020-03039-y

2. Fu J, Wang H. Precision diagnosis and treatment of liver cancer in China. Cancer Lett. 2018;412:283-288. doi:10.1016/j.canlet.2017.10.008

3. Tabrizian P, Jibara G, Shrager B, Schwartz M, Roayaie S. Recurrence of hepatocellular cancer after resection: patterns, treatments, and prognosis. Ann Surg. 2015;261(5):947-955. doi:10.1097/SLA.0000000000000710

4. Zhu K, Huang J, Lai L, et al. Medium or large hepatocellular carcinoma: sorafenib combined with transarterial chemoembolization and radiofrequency ablation. Radiology. 2018;288(1):300-307. doi:10.1148/radiol.2018172028

5. Wei L, Wang X, Lv L, et al. The emerging role of microRNAs and long noncoding RNAs in drug resistance of hepatocellular carcinoma. Mol Cancer. 2019;18(1):147. doi:10.1186/s12943-019-1086-z

6. Zhou XT, Ding J, Li HY, et al. Hedgehog signalling mediates drug resistance through targeting TAP1 in hepatocellular carcinoma. J Cell Mol Med. 2020;24(7):4298-4311. doi:10.1111/jcmm.15090

7. Tang Z, He J, Zou J, Yu S, Sun X, Qin L. Cisplatin-resistant HepG2 cell-derived exosomes transfer cisplatin resistance to cisplatin-sensitive cells in HCC. PeerJ. 2021;9:e11200. doi:10.7717/peerj.11200

8. Bruix J, Qin S, Merle P, et al. Regorafenib for patients with hepatocellular carcinoma who progressed on sorafenib treatment (RESORCE): a randomised, double-blind, placebo-controlled, Phase 3 trial. Lancet. 2017;389(10064):56-66. doi:10.1016/S0140-6736(16)32453-9

9. Lal CV, Olave N, Travers C, et al. Exosomal microRNA predicts and protects against severe bronchopulmonary dysplasia in extremely premature infants. JCI Insight. 2018;3(5). doi:10.1172/jci.insight.93994

10. Takasugi M. Emerging roles of extracellular vesicles in cellular senescence and aging. Aging Cell. 2018;17(2):e12734. doi:10.1111/acel.12734

11. De la fuente A, Alonso-Alconada L, Costa C, et al. M-trap: exosome-based capture of tumor cells as a new technology in peritoneal metastasis. $J$ Natl Cancer Inst. 2015;107(9):djv184. doi:10.1093/jnci/djv184

12. Wang J, Yeung BZ, Cui M, et al. Exosome is a mechanism of intercellular drug transfer: application of quantitative pharmacology. $J$ Control Release. 2017;268:147-158. doi:10.1016/j.jconrel.2017.10.020

13. Li C, Hou X, Zhang P, et al. Exosome-based tumor therapy: opportunities and challenges. Curr Drug Metab. 2020;21(5):339-351. doi:10.2174/ 1389200221666200515103354

14. Giallombardo M, Taverna S, Alessandro R, Hong D, Rolfo C. Exosome-mediated drug resistance in cancer: the near future is here. Ther Adv Med Oncol. 2016;8(5):320-322. doi:10.1177/1758834016648276 
15. Li R, Dong C, Jiang K, et al. Rab27B enhances drug resistance in hepatocellular carcinoma by promoting exosome-mediated drug efflux. Carcinogenesis. 2020;41(11):1583-1591. doi:10.1093/carcin/bgaa029

16. Chinnappan M, Srivastava A, Amreddy N, et al. Exosomes as drug delivery vehicle and contributor of resistance to anticancer drugs. Cancer Lett. 2020;486:18-28. doi:10.1016/j.canlet.2020.05.004

17. Yukawa H, Suzuki K, Aoki K, et al. Imaging of angiogenesis of human umbilical vein endothelial cells by uptake of exosomes secreted from hepatocellular carcinoma cells. Sci Rep. 2018;8(1):6765. doi:10.1038/s41598-018-24563-0

18. Nault JC, Villanueva A. Intratumor molecular and phenotypic diversity in hepatocellular carcinoma. Clin Cancer Res. 2015;21(8):1786-1788. doi:10.1158/1078-0432.CCR-14-2602

19. Li C, Xu X. Biological functions and clinical applications of exosomal non-coding RNAs in hepatocellular carcinoma. Cell Mol Life Sci. 2019;76(21):4203-4219. doi:10.1007/s00018-019-03215-0

20. Fu X, Liu M, Qu S, et al. Exosomal microRNA-32-5p induces multidrug resistance in hepatocellular carcinoma via the PI3K/Akt pathway. $J$ Exp Clin Cancer Res. 2018;37(1):52. doi:10.1186/s13046-018-0677-7

21. Ma J, Zeng S, Zhang Y, et al. BMP4 promotes oxaliplatin resistance by an induction of epithelial-mesenchymal transition via MEK1/ERK/ ELK1 signaling in hepatocellular carcinoma. Cancer Lett. 2017;411:117-129. doi:10.1016/j.canlet.2017.09.041

22. Marin JJG, Macias RIR, Monte MJ, et al. Molecular bases of drug resistance in hepatocellular carcinoma. Cancers (Basel). 2020;12(6):1663.

23. Baig MS, Roy A, Rajpoot S, et al. Tumor-derived exosomes in the regulation of macrophage polarization. Inflamm Res. 2020;69(5):435-451. doi:10.1007/s00011-020-01318-0

24. Dong X, Bai X, Ni J, et al. Exosomes and breast cancer drug resistance. Cell Death Dis. 2020;11(11):987. doi:10.1038/s41419-020-03189-Z

25. Shedden K, Xie XT, Chandaroy P, Chang YT, Rosania GR. Expulsion of small molecules in vesicles shed by cancer cells: association with gene expression and chemosensitivity profiles. Cancer Res. 2003;63(15):4331-4337.

26. Safaei R, Larson BJ, Cheng TC, et al. Abnormal lysosomal trafficking and enhanced exosomal export of cisplatin in drug-resistant human ovarian carcinoma cells. Mol Cancer Ther. 2005;4(10):1595-1604. doi:10.1158/1535-7163.MCT-05-0102

27. Steinbichler TB, Dudas J, Skvortsov S, Ganswindt U, Riechelmann H, Skvortsova II. Therapy resistance mediated by exosomes. Mol Cancer. 2019;18(1):58. doi:10.1186/s12943-019-0970-x

28. Januchowski R, Sterzynska K, Zaorska K, et al. Analysis of MDR genes expression and cross-resistance in eight drug resistant ovarian cancer cell lines. J Ovarian Res. 2016;9(1):65. doi:10.1186/s13048-016-0278-z

29. Namisaki T, Schaeffeler E, Fukui H, et al. Differential expression of drug uptake and efflux transporters in Japanese patients with hepatocellular carcinoma. Drug Metab Dispos. 2014;42(12):2033-2040. doi:10.1124/dmd.114.059832

30. Huttunen J, Gynther M, Huttunen KM. Targeted efflux transporter inhibitors - A solution to improve poor cellular accumulation of anti-cancer agents. Int J Pharm. 2018;550(1-2):278-289. doi:10.1016/j.ijpharm.2018.08.047

31. Wang X, Qiao D, Chen L, et al. Chemotherapeutic drugs stimulate the release and recycling of extracellular vesicles to assist cancer cells in developing an urgent chemoresistance. Mol Cancer. 2019;18(1):182. doi:10.1186/s12943-019-1114-z

32. Koch R, Aung T, Vogel D, et al. Nuclear trapping through inhibition of exosomal export by indomethacin increases cytostatic efficacy of doxorubicin and pixantrone. Clin Cancer Res. 2016;22(2):395-404. doi:10.1158/1078-0432.CCR-15-0577

33. Xu Y, Qiu A, Peng F, Tan X, Wang J, Gong X. Exosomal transfer of circular RNA FBXW7 ameliorates the chemoresistance to oxaliplatin in colorectal cancer by sponging miR-18b-5p. Neoplasma. 2021;68(1):108-118. doi:10.4149/neo_2020_200417N414

34. Wang Y, Wang Y, Qin Z, et al. The role of non-coding RNAs in ABC transporters regulation and their clinical implications of multidrug resistance in cancer. Expert Opin Drug Metab Toxicol. 2021;17(3):291-306. doi:10.1080/17425255.2021.1887139

35. Borel F, Han R, Visser A, et al. Adenosine triphosphate-binding cassette transporter genes up-regulation in untreated hepatocellular carcinoma is mediated by cellular microRNAs. Hepatology. 2012;55(3):821-832. doi:10.1002/hep.24682

36. Takahashi K, Yan IK, Wood J, Haga H, Patel T. Involvement of extracellular vesicle long noncoding RNA (linc-VLDLR) in tumor cell responses to chemotherapy. Mol Cancer Res. 2014;12(10):1377-1387. doi:10.1158/1541-7786.MCR-13-0636

37. Ding C, Yi X, Wu X, et al. Exosome-mediated transfer of circRNA CircNFIX enhances temozolomide resistance in glioma. Cancer Lett. 2020;479:1-12. doi:10.1016/j.canlet.2020.03.002

38. Milane L, Singh A, Mattheolabakis G, Suresh M, Amiji MM. Exosome mediated communication within the tumor microenvironment. $J$ Control Release. 2015;219:278-294. doi:10.1016/j.jconrel.2015.06.029

39. Yang N, Li S, Li G, et al. The role of extracellular vesicles in mediating progression, metastasis and potential treatment of hepatocellular carcinoma. Oncotarget. 2017;8(2):3683-3695. doi:10.18632/oncotarget.12465

40. Cao LQ, Yang XW, Chen YB, Zhang DW, Jiang XF, Xue P. Exosomal miR-21 regulates the TETs/PTENp1/PTEN pathway to promote hepatocellular carcinoma growth. Mol Cancer. 2019;18(1):148. doi:10.1186/s12943-019-1075-2

41. Takahashi K, Yan IK, Kogure T, Haga H, Patel T. Extracellular vesicle-mediated transfer of long non-coding RNA ROR modulates chemosensitivity in human hepatocellular cancer. FEBS Open Bio. 2014;4:458-467. doi:10.1016/j.fob.2014.04.007

42. Hansen TB, Jensen TI, Clausen BH, et al. Natural RNA circles function as efficient microRNA sponges. Nature. 2013;495(7441):384-388. doi:10.1038/nature11993

43. Xu J, Ji L, Liang Y, et al. CircRNA-SORE mediates sorafenib resistance in hepatocellular carcinoma by stabilizing YBX1. Signal Transduct Target Ther. 2020;5(1):298. doi:10.1038/s41392-020-00375-5

44. Qu Z, Wu J, Wu J, Luo D, Jiang C, Ding Y. Exosomes derived from HCC cells induce sorafenib resistance in hepatocellular carcinoma both in vivo and in vitro. $J$ Exp Clin Cancer Res. 2016;35(1):159. doi:10.1186/s13046-016-0430-z

45. Wang X, Zhang H, Bai M, et al. Exosomes serve as nanoparticles to deliver anti-miR-214 to reverse chemoresistance to cisplatin in gastric cancer. Mol Ther. 2018;26(3):774-783. doi:10.1016/j.ymthe.2018.01.001

46. Zhang K, Shao CX, Zhu JD, et al. Exosomes function as nanoparticles to transfer miR-199a-3p to reverse chemoresistance to cisplatin in hepatocellular carcinoma. Biosci Rep. 2020;40(7):BSR20194026.

47. Lou G, Song X, Yang F, et al. Exosomes derived from miR-122-modified adipose tissue-derived MSCs increase chemosensitivity of hepatocellular carcinoma. J Hematol Oncol. 2015;8:122. doi:10.1186/s13045-015-0220-7

48. Bai Y, Ding M, Lu D, et al. Long noncoding RNA AC007639.1 promotes the pathogenesis and progression of hepatocellular carcinoma through inhibiting apoptosis and stimulating chemotherapeutic resistance. Front Oncol. 2021;11:715541. doi:10.3389/fonc.2021.715541 
49. Sangro B, Sarobe P, Hervas-Stubbs S, Melero I. Advances in immunotherapy for hepatocellular carcinoma. Nat Rev Gastroenterol Hepatol. 2021;18(8):525-543. doi:10.1038/s41575-021-00438-0

50. Pfister D, Nunez NG, Pinyol R, et al. NASH limits anti-tumour surveillance in immunotherapy-treated HCC. Nature. 2021;592(7854):450-456. doi:10.1038/s41586-021-03362-0

51. Yi C, Chen L, Lin Z, et al. Lenvatinib targets FGF receptor 4 to enhance antitumor immune response of anti-programmed cell death-1 in HCC. Hepatology. 2021;74(5):2544-2560. doi:10.1002/hep.31921

52. Ruf B, Heinrich B, Greten TF. Immunobiology and immunotherapy of HCC: spotlight on innate and innate-like immune cells. Cell Mol Immunol. 2021;18(1):112-127. doi:10.1038/s41423-020-00572-w

53. Han Q, Zhao H, Jiang Y, Yin C, Zhang J. HCC-derived exosomes: critical player and target for cancer immune escape. Cells. 2019;8(6). doi:10.3390/cells8060558

54. Zhang PF, Gao C, Huang XY, et al. Cancer cell-derived exosomal circUHRF1 induces natural killer cell exhaustion and may cause resistance to anti-PD1 therapy in hepatocellular carcinoma. Mol Cancer. 2020;19(1):110. doi:10.1186/s12943-020-01222-5

55. Ye L, Zhang Q, Cheng Y, et al. Tumor-derived exosomal HMGB1 fosters hepatocellular carcinoma immune evasion by promoting TIM-1(+) regulatory B cell expansion. J Immunother Cancer. 2018;6(1):145. doi:10.1186/s40425-018-0451-6

56. Yin C, Han Q, Xu D, Zheng B, Zhao X, Zhang J. SALL4-mediated upregulation of exosomal miR-146a-5p drives T-cell exhaustion by M2 tumor-associated macrophages in HCC. Oncoimmunology. 2019;8(7):1601479. doi:10.1080/2162402X.2019.1601479

57. Morrissey SM, Yan J. Exosomal PD-L1: roles in tumor progression and immunotherapy. Trends Cancer. 2020;6(7):550-558. doi:10.1016/j. trecan.2020.03.002

58. Ayala-Mar S, Donoso-Quezada J, Gonzalez-Valdez J. Clinical implications of exosomal PD-L1 in cancer immunotherapy. J Immunol Res. 2021;2021:8839978. doi:10.1155/2021/8839978

59. Chen G, Huang AC, Zhang W, et al. Exosomal PD-L1 contributes to immunosuppression and is associated with anti-PD-1 response. Nature. 2018;560(7718):382-386. doi:10.1038/s41586-018-0392-8

60. Xu Z, Zeng S, Gong Z, Yan Y. Exosome-based immunotherapy: a promising approach for cancer treatment. Mol Cancer. 2020;19(1):160. doi:10.1186/s12943-020-01278-3

61. Lv LH, Wan YL, Lin Y, et al. Anticancer drugs cause release of exosomes with heat shock proteins from human hepatocellular carcinoma cells that elicit effective natural killer cell antitumor responses in vitro. J Biol Chem. 2012;287(19):15874-15885. doi:10.1074/jbc.M112.340588

62. Zhou G, Sprengers D, Mancham S, et al. Reduction of immunosuppressive tumor microenvironment in cholangiocarcinoma by ex vivo targeting immune checkpoint molecules. $J$ Hepatol. 2019;71(4):753-762. doi:10.1016/j.jhep.2019.05.026

63. Xu R, Rai A, Chen M, Suwakulsiri W, Greening DW, Simpson RJ. Extracellular vesicles in cancer - implications for future improvements in cancer care. Nat Rev Clin Oncol. 2018;15(10):617-638. doi:10.1038/s41571-018-0036-9

64. Cheng HS, Lee JXT, Wahli W, Tan NS. Exploiting vulnerabilities of cancer by targeting nuclear receptors of stromal cells in tumor microenvironment. Mol Cancer. 2019;18(1):51. doi:10.1186/s12943-019-0971-9

65. Tian XP, Wang CY, Jin XH, et al. Acidic microenvironment up-regulates exosomal miR-21 and miR-10b in early-stage hepatocellular carcinoma to promote cancer cell proliferation and metastasis. Theranostics. 2019;9(7):1965-1979. doi:10.7150/thno.30958

66. Pascut D, Pratama MY, Vo NVT, Masadah R, Tiribelli C. The crosstalk between tumor cells and the microenvironment in hepatocellular carcinoma: the role of exosomal microRNAs and their clinical implications. Cancers (Basel). 2020;12(4):823. doi:10.3390/cancers12040823

67. Biswas S, Mandal G, Roy Chowdhury S, et al. Exosomes produced by mesenchymal stem cells drive differentiation of myeloid cells into immunosuppressive M2-polarized macrophages in breast cancer. J Immunol. 2019;203(12):3447-3460. doi:10.4049/jimmunol.1900692

68. Zhang B, Yeo RWY, Lai RC, Sim EWK, Chin KC, Lim SK. Mesenchymal stromal cell exosome-enhanced regulatory T-cell production through an antigen-presenting cell-mediated pathway. Cytotherapy. 2018;20(5):687-696. doi:10.1016/j.jcyt.2018.02.372

69. Santos P, Almeida F. Role of exosomal miRNAs and the tumor microenvironment in drug resistance. Cells. 2020;9(6):1450. doi:10.3390/ cells 9061450

70. Zhou Y, Ren H, Dai B, et al. Hepatocellular carcinoma-derived exosomal miRNA-21 contributes to tumor progression by converting hepatocyte stellate cells to cancer-associated fibroblasts. J Exp Clin Cancer Res. 2018;37(1):324. doi:10.1186/s13046-018-0965-2

71. Fang T, Lv H, Lv G, et al. Tumor-derived exosomal miR-1247-3p induces cancer-associated fibroblast activation to foster lung metastasis of liver cancer. Nat Commun. 2018;9(1):191. doi:10.1038/s41467-017-02583-0

72. Zhang H, Deng T, Liu R, et al. CAF secreted miR-522 suppresses ferroptosis and promotes acquired chemo-resistance in gastric cancer. Mol Cancer. 2020;19(1):43. doi:10.1186/s12943-020-01168-8

73. Duan H, Liu Y, Gao Z, Huang W. Recent advances in drug delivery systems for targeting cancer stem cells. Acta Pharm Sin B. 2021;11(1):5570. doi:10.1016/j.apsb.2020.09.016

74. Li W, Zhang L, Guo B, et al. Exosomal FMR1-AS1 facilitates maintaining cancer stem-like cell dynamic equilibrium via TLR7/NFkappaB/cMyc signaling in female esophageal carcinoma. Mol Cancer. 2019;18(1):22. doi:10.1186/s12943-019-0949-7

75. Lee NK, Kothandan VK, Kothandan S, Byun Y, Hwang SR. Exosomes and cancer stem cells in cancer immunity: current reports and future directions. Vaccines (Basel). 2021;9(5). doi:10.3390/vaccines9050441

76. Yang Z, Zhao N, Cui J, Wu H, Xiong J, Peng T. Exosomes derived from cancer stem cells of gemcitabine-resistant pancreatic cancer cells enhance drug resistance by delivering miR-210. Cell Oncol (Dordr). 2020;43(1):123-136. doi:10.1007/s13402-019-00476-6

77. Li W, Xin X, Li X, Geng J, Sun Y. Exosomes secreted by M2 macrophages promote cancer stemness of hepatocellular carcinoma via the miR27a-3p/TXNIP pathways. Int Immunopharmacol. 2021;101(Pt A):107585. doi:10.1016/j.intimp.2021.107585

78. Blackwell RH, Foreman KE, Gupta GN. The role of cancer-derived exosomes in tumorigenicity \& epithelial-to-mesenchymal transition. Cancers (Basel). 2017;9(8):105. doi:10.3390/cancers9080105

79. Abudoureyimu M, Zhou H, Zhi Y, et al. Recent progress in the emerging role of exosome in hepatocellular carcinoma. Cell Prolif. 2019;52(2): e12541. doi:10.1111/cpr.12541

80. Panda M, Tripathi SK, Biswal BK. SOX9: an emerging driving factor from cancer progression to drug resistance. Biochim Biophys Acta Rev Cancer. 2021;1875(2):188517. doi:10.1016/j.bbcan.2021.188517

81. Singh A, Settleman J. EMT, cancer stem cells and drug resistance: an emerging axis of evil in the war on cancer. Oncogene. 2010;29(34):47414751. doi:10.1038/onc. 2010.215 
82. Brozovic A. The relationship between platinum drug resistance and epithelial-mesenchymal transition. Arch Toxicol. 2017;91(2):605-619. doi:10.1007/s00204-016-1912-7

83. Santos JC, Lima NDS, Sarian LO, Matheu A, Ribeiro ML, Derchain SFM. Exosome-mediated breast cancer chemoresistance via miR-155 transfer. Sci Rep. 2018;8(1):829. doi:10.1038/s41598-018-19339-5

84. Hisakane K, Seike M, Sugano T, et al. Exosome-derived miR-210 involved in resistance to osimertinib and epithelial-mesenchymal transition in EGFR mutant non-small cell lung cancer cells. Thorac Cancer. 2021;12(11):1690-1698. doi:10.1111/1759-7714.13943

85. Kim HS, Kim JS, Park NR, et al. Exosomal miR-125b exerts anti-metastatic properties and predicts early metastasis of hepatocellular carcinoma. Front Oncol. 2021;11:637247. doi:10.3389/fonc.2021.637247

86. Liu T, Zhang X, Du L, et al. Exosome-transmitted miR-128-3p increase chemosensitivity of oxaliplatin-resistant colorectal cancer. Mol Cancer. 2019;18(1):43. doi:10.1186/s12943-019-0981-7

87. Qian F, Wang J, Wang Y, et al. MiR-378a-3p as a putative biomarker for hepatocellular carcinoma diagnosis and prognosis: computational screening with experimental validation. Clin Transl Med. 2021;11(2):e307. doi:10.1002/ctm2.307

88. Yu L, Kim J, Jiang L, et al. MTR4 drives liver tumorigenesis by promoting cancer metabolic switch through alternative splicing. Nat Commun. 2020;11(1):708. doi:10.1038/s41467-020-14437-3

89. Myojin Y, Kodama T, Maesaka K, et al. ST6GAL1 is a novel serum biomarker for lenvatinib-susceptible FGF19-driven hepatocellular carcinoma. Clin Cancer Res. 2021;27(4):1150-1161. doi:10.1158/1078-0432.CCR-20-3382

90. Vidal M. Exosomes and GPI-anchored proteins: judicious pairs for investigating biomarkers from body fluids. Adv Drug Deliv Rev. 2020;161162:110-123. doi:10.1016/j.addr.2020.08.006

91. Zhu J, Tang B, Gao Y, et al. Predictive models for HCC prognosis, recurrence risk, and immune infiltration based on two exosomal genes: MYL6B and THOC2. J Inflamm Res. 2021;14:4089-4109. doi:10.2147/JIR.S315957

92. Hoshino A, Kim HS, Bojmar L, et al. Extracellular vesicle and particle biomarkers define multiple human cancers. Cell. 2020;182(4):10441061 e1018. doi:10.1016/j.cell.2020.07.009

93. Du J, Wan Z, Wang C, et al. Designer exosomes for targeted and efficient ferroptosis induction in cancer via chemo-photodynamic therapy. Theranostics. 2021;11(17):8185-8196. doi:10.7150/thno.59121

94. Zhao X, Wu D, Ma X, Wang J, Hou W, Zhang W. Exosomes as drug carriers for cancer therapy and challenges regarding exosome uptake. Biomed Pharmacother. 2020;128:110237. doi:10.1016/j.biopha.2020.110237

95. Liu J, Ye Z, Xiang M, et al. Functional extracellular vesicles engineered with lipid-grafted hyaluronic acid effectively reverse cancer drug resistance. Biomaterials. 2019;223:119475. doi:10.1016/j.biomaterials.2019.119475

96. Fan Z, Xiao K, Lin J, Liao Y, Huang X. Functionalized DNA enables programming exosomes/vesicles for tumor imaging and therapy. Small. 2019;15(47):e1903761. doi:10.1002/smll.201903761

97. Lin D, Zhang H, Liu R, et al. iRGD-modified exosomes effectively deliver CPT1A siRNA to colon cancer cells, reversing oxaliplatin resistance by regulating fatty acid oxidation. Mol Oncol. 2021;15:3430-3446. doi:10.1002/1878-0261.13052

98. Lima LG, Ham S, Shin H, et al. Tumor microenvironmental cytokines bound to cancer exosomes determine uptake by cytokine receptorexpressing cells and biodistribution. Nat Commun. 2021;12(1):3543. doi:10.1038/s41467-021-23946-8

99. Zhang X, Ng HLH, Lu A, et al. Drug delivery system targeting advanced hepatocellular carcinoma: current and future. Nanomedicine. 2016;12 (4):853-869. doi:10.1016/j.nano.2015.12.381

100. Farinha D, Migawa M, Sarmento-Ribeiro A, Faneca H. A combined antitumor strategy mediated by a new targeted nanosystem to hepatocellular carcinoma. Int J Nanomedicine. 2021;16:3385-3405. doi:10.2147/IJN.S302288

101. Guo J, Yu Z, Sun D, Zou Y, Liu Y, Huang L. Two nanoformulations induce reactive oxygen species and immunogenetic cell death for synergistic chemo-immunotherapy eradicating colorectal cancer and hepatocellular carcinoma. Mol Cancer. 2021;20(1):10. doi:10.1186/ s12943-020-01297-0

102. Liu X, Sun Y, Xu S, et al. Homotypic cell membrane-cloaked biomimetic nanocarrier for the targeted chemotherapy of hepatocellular carcinoma. Theranostics. 2019;9(20):5828-5838. doi:10.7150/thno.34837

103. Moore C, Kosgodage U, Lange S, Inal JM. The emerging role of exosome and microvesicle- (EMV-) based cancer therapeutics and immunotherapy. Int $J$ Cancer. 2017;141(3):428-436. doi:10.1002/ijc.30672

104. Lu Z, Zuo B, Jing R, et al. Dendritic cell-derived exosomes elicit tumor regression in autochthonous hepatocellular carcinoma mouse models. $J$ Hepatol. 2017;67(4):739-748. doi:10.1016/j.jhep.2017.05.019

105. Li J, Huang S, Zhou Z, et al. Exosomes derived from rAAV/AFP-transfected dendritic cells elicit specific T cell-mediated immune responses against hepatocellular carcinoma. Cancer Manag Res. 2018;10:4945-4957. doi:10.2147/CMAR.S178326

106. Balkwill FR, Capasso M, Hagemann T. The tumor microenvironment at a glance. J Cell Sci. 2012;125(Pt 23):5591-5596. doi:10.1242/ jcs. 116392

107. Garofalo M, Di Leva G, Romano G, et al. miR-221\&222 regulate TRAIL resistance and enhance tumorigenicity through PTEN and TIMP3 downregulation. Cancer Cell. 2009;16(6):498-509. doi:10.1016/j.ccr.2009.10.014

108. Zhao Y, Qi XM, Chen J, et al. The miR-491-3p/Sp3/ABCB1 axis attenuates multidrug resistance of hepatocellular carcinoma. Cancer Lett. 2017;408:102-111. doi:10.1016/j.canlet.2017.08.027

109. Zheng SZ, Sun P, Wang JP, et al. MiR-34a overexpression enhances the inhibitory effect of doxorubicin on HepG2 cells. World J Gastroenterol. 2019;25(22):2752-2762. doi:10.3748/wjg.v25.i22.2752

110. Ma J, Wang T, Guo R, et al. Involvement of miR-133a and miR-326 in ADM resistance of HepG2 through modulating expression of ABCC1. $J$ Drug Target. 2015;23(6):519-524. doi:10.3109/1061186X.2015.1015536

111. Wang XJ, Zhang DL, Fu C, et al. MiR-183 modulates multi-drug resistance in hepatocellular cancer (HCC) cells via miR-183-IDH2 /SOCS6HIF-1 alpha feedback loop. Eur Rev Med Pharmacol Sci. 2016;20(10):2020-2027.

112. Kong J, Qiu Y, Li Y, et al. TGF-betal elevates P-gp and BCRP in hepatocellular carcinoma through HOTAIR/miR-145 axis. Biopharm Drug Dispos. 2019;40(2):70-80. doi:10.1002/bdd.2172

113. Hu HQ, Yang L, Li LJ, et al. Long non-coding RNA KCNQ1OT1 modulates oxaliplatin resistance in hepatocellular carcinoma through miR-75p/ABCC1 axis. Biochem Biophys Res Commun. 2018;503(4):2400-2406. doi:10.1016/j.bbrc.2018.06.168 
114. Huang W, Huang F, Feng C. CircFoxo3 promotes Adriamycin resistance through regulation of miR-199a-5p/ATP binding cassette subfamily C member 1 axis in hepatocellular carcinoma. Onco Targets Ther. 2020;13:5113-5122. doi:10.2147/OTT.S243571

115. Hou Y, Yu Z, Tam NL, et al. Exosome-related lncRNAs as predictors of HCC patient survival: a prognostic model. Am J Transl Res. 2018;10 (6):1648-1662.

\section{Publish your work in this journal}

The Journal of Hepatocellular Carcinoma is an international, peer-reviewed, open access journal that offers a platform for the dissemination and study of clinical, translational and basic research findings in this rapidly developing field. Development in areas including, but not limited to, epidemiology, vaccination, hepatitis therapy, pathology and molecular tumor classification and prognostication are all considered for publication. The manuscript management system is completely online and includes a very quick and fair peer-review system, which is all easy to use. Visit http://www.dovepress.com/testimonials.php to read real quotes from published authors.

Submit your manuscript here: https://www.dovepress.com/journal-of-hepatocellular-carcinoma-journal 